\title{
Multi-agent flocking under general communication rule
}

\author{
Samuel Martin, Antoine Girard, Arastoo Fazeli and Ali Jadbabaie
}

\begin{abstract}
In this paper, we consider a multi-agent system consisting of mobile agents with second-order dynamics. The communication network is determined by a general interaction rule based on the distance between agents. The goal of this paper is to determine practical conditions ensuring that the agents asymptotically agree on a common velocity, i.e. a flocking behavior is achieved. Unlike previous studies on the topic, our results simultaneously satisfy the three following features: our conditions apply to a model which does not require long distance communication; they only depend on the initial positions and velocities of the agents, and most importantly, our results allow for the disconnection of communication links which are not necessary for flocking. To circumvent the difficulty arising from the state dependent dynamics, a suitable bounding process is used. We apply our result to two cases where communication takes place either within deterministic or stochastic distance radiuses. Our result is illustrated through simulations.
\end{abstract}

\section{INTRODUCTION}

Analysis and design of cooperative behaviors in networked dynamic systems has lately received a lot of attention. Multiagent systems find applications in technical areas such as mobile sensor networks [CB09], cooperative robotics [CMKB04] or distributed implementation of algorithms [TBA86]. A central question arising in the study of multi-agent systems is whether the group will be able to reach a consensus. Intuitively, agents are said to reach a consensus when all individuals agree on a common value (e.g. the heading direction of a flock of birds, the candidate to elect for voters).

To carry out formal studies on consensus problems, one usually assumes that the multi-agent system follows some abstract communication protocol and then investigates conditions under which a consensus will be reached. Existing frameworks include discrete and continuous-time systems involving or neglecting time-delays in the communication process (see e.g. [OSFM07]). The communication network between agents is usually modeled by a graph. The graph topology is either assumed to be fixed or can switch over time. The switching topology of the interactions is sometimes assumed to depend on the state of the agents (e.g. the strength of the communication can be a function of the distance between agents).

Consensus can be modeled in a deterministic fashion; however, in many applications it seems that the topology of the network is quite random. Recently there has been a growing interest in studying consensus algorithms in a probabilistic setting [HM05], [Wu06], [PS07], [TSJ08], [PT07], [AOP10], [TN10], [Cha09], [JLM03], [TJP07], where network

This research has been partially supported by the ANR (project VEDECY). S. Martin and A. Girard are with the Laboratoire Jean Kuntzmann, Université de Grenoble, B.P. 53, 38041 Grenoble Cedex 9, France. samuel.martinsa@gmail.com. A. Fazeli and A. Jadbabaie are with the Department of Electrical and Systems Engineering at University of Pennsylvania. changes can be independent, identically distributed (i.i.d.) over time [TSJ08], ergodic-stationary [TSJ10], or Markovian [IJ09]. This randomness can be due to the unpredictability of the environment in which the communication between agents occurs or due to the inherent probabilistic characteristic of the communication among agents [FZ07].

An example where consensus protocols apply is when agents in the network communicate if they are within some distance which is governed by some random process. For instance in wireless networks, the log-normal shadowing statistical model can be approximated assuming that the range within which agents communicate is random and uniformly distributed over some interval [BW09]. Therefore, in these probabilistic cases one should investigate conditions under which consensus will be reached with some given probability. It would be quite useful if a lower bound for this probability could be found as well.

Many papers have investigated sufficient conditions ensuring asymptotic consensus. The assumptions made in the models are usually rather general (see e.g. [Mor05]). This enables the given conditions to apply in a wide range of cases. Conditions usually require some connectivity properties on the expected communication network to hold over time. A drawback in such conditions is that they often cannot be verified a priori.

In this paper we consider a group of agents with second order dynamics. We generalize previous works from Martin and Girard [MG10] and Martin et al. [MFJG12] by considering the following setting : The communication network is determined by an interaction rule based on the distance between agents (see section II). The influence between two agents is a non-increasing function of the distance between them. This influence is supposed to be symmetric. Under these mild assumptions, the goal of the paper is to determine practical conditions (on the initial positions and velocities of agents) ensuring that the agents eventually agree on a common velocity, i.e. a flocking behavior is achieved (see section IV-A). These conditions depend on the initial configuration only. As defined, the dynamics is state dependent and therefore hard to forecast. We exhibit a suitable bounding process which allows us to build upon previous work such as [TSJ06], to establish such conditions (see section IV-C). In order to show the applicability of our theoretical result, we apply it to two distinct communication rules. First, agents interact whenever their distance is smaller than a fixed communication radius (section V-A). Then, we set distinct communication radiuses for each agents, each of them varying randomly in time (section V-A). In the latter case, we show how to compute the optimal bound for flocking (section VI-A). This allows us to test our result in numerical simulations (section VI-B). Finally, we investigate the impact of the mean and variance 
of the communication radiuses on the bound for flocking (section VI-B). Our result is illustrated through simulations.

Unlike previous studies on the topic, our results simultaneously satisfy the following features:

- the change in the network topology is endogenous, i.e. it depends explicitly on the agents'states,

- our conditions apply to a model which does not require long distance communication,

- the conditions only depend on the initial configuration of the system,

- and most importantly, our results allow for the disconnection of communication links which are not necessary in order to obtain asymptotic flocking.

Related results in the literature include [DM09] where the authors assume a hierarchy in the communication network and [CM08] where the authors consider additive noise to the dynamics (which makes the asymptotic velocity alignment impossible to achieve). Another viewpoints is adopted in [ZTJP09] where authors control the communication weights in order to preserve connectivity.

\section{Problem Formulation}

In this paper, we study a discrete time, multi-agent system. We consider a set $\mathcal{N}=\{1, \ldots, n\}$ of mobile agents evolving in a $d$-dimensional space. Each agent $i \in \mathcal{N}$ is characterized by its position $x_{i}(t) \in \mathbb{R}^{d}$ and its velocity $v_{i}(t) \in \mathbb{R}^{d}$ at time $\varepsilon t$ where $\varepsilon>0$ is the time step parameter of the system. The initial positions and velocities are given by $x_{i}(0)=x_{i}^{0}$ and $v_{i}(0)=v_{i}^{0}$. The agents exchange information over a communication network. The topology of the network depends on the relative position of agents and is therefore subject to change. The agents use the available information to adapt their velocity in order to achieve a flocking behavior. Formally, the evolution of each agent $i \in \mathcal{N}$ occurs every time step $\varepsilon$ and for some $t \in \mathbb{N}$, this evolution is described at time $\varepsilon t$ by the following discrete-time protocol:

$$
\begin{aligned}
& x_{i}(t+1)=x_{i}(t)+\varepsilon v_{i}(t) \\
& v_{i}(t+1)=v_{i}(t)+\varepsilon \sum_{j \in \mathcal{N}} a_{i j}\left(t,\left\|x_{j}(t)-x_{i}(t)\right\|\right)\left(v_{j}(t)-v_{i}(t)\right) .
\end{aligned}
$$

where $a_{i j}\left(t,\left\|x_{j}(t)-x_{i}(t)\right\|\right) \geq 0$ is the communication weight between $i$ and $j$ and $\|$.$\| denotes the Euclidean norm { }^{1}$. The weight $a_{i j}\left(t,\left\|x_{j}(t)-x_{i}(t)\right\|\right)>0$ when communication between agents $j$ and $i$ takes place at time $\varepsilon t$ and is equal to 0 otherwise.

In this paper we focus on communication of agents defined by a metric rule, which explains why the communication weights depend explicitly on the distance between agents. In this paper, we also assume that the following condition is satisfied:

Assumption 1. The communication weights $a_{i j}$ are symmetric and decrease when agents go away from one another : for all $i, j \in \mathcal{N}$, and for all $t \in \mathbb{R}^{+}$,

$$
\left\{\begin{array}{l}
\forall z \in \mathbb{R}^{+}, a_{i j}(t, z)=a_{j i}(t, z), \\
\forall z_{1}, z_{2} \in \mathbb{R}^{+}, z_{1} \leq z_{2} \Rightarrow a_{i j}\left(t, z_{1}\right) \geq a_{i j}\left(t, z_{2}\right) .
\end{array}\right.
$$

\footnotetext{
${ }^{1}$ In the following, $\|$.$\| will denote the usual Euclidean norm on \mathbb{R}^{d}$ or $\mathbb{R}^{n d}$ depending on the context.
}

The symmetry assumption allows us to make use of algebraic graph theory. Without this assumption our main results do not hold. The fact that the weights decrease with the distance between agents reflects communication constraints often found in real systems: it is easier for the agents to sense each other when they are closer to one another. As we shall see in section $\mathrm{V}$-B, the fact that the weights $a_{i j}$ depends explicitly on time allows to include important classes of systems such as systems with disturbance. Metric interactions are usually assumed to be a good representation of how collective behavior takes place. Thus, most of the literature

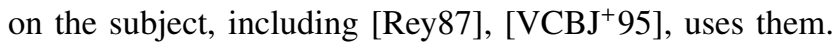

We say that the agents achieve a flocking behavior if all the agents asymptotically move with a common direction:

$$
\forall i, j \in \mathcal{N}, \lim _{t \rightarrow+\infty}\left|v_{i}(t)-v_{j}(t)\right|=0 .
$$

The goal of this paper is to determine sufficient conditions which guarantee that the flocking behavior is achieved. Such conditions should be easily verifiable using only the initial positions and velocities of agents.

\section{Preliminaries}

In this section, we review some results from algebraic graph theory and multi-agent systems that will be useful in the subsequent discussion.

\section{A. Algebraic Graph Theory}

Let us recall some standard results from algebraic graph theory. More details can be found, for instance, in [GR01], [Mer94]. We then apply the algebraic formalism to system (1) to obtain preliminary results. A similar approach was carried out in [TSJ06] and [KM07].

Denote the communication weight matrix $A(t)=\left[a_{i j}(t)\right]$. We only consider the case where $A$ is symmetric with non-negative entries according to the definition of the communication weights. For the rest of the section, we drop the dependence on time. Let $D=\operatorname{diag}\left(d_{1}, d_{2}, \ldots, d_{n}\right)$ be the degree matrix of $A$ where $d_{i}=\sum_{j \in \mathcal{N}} a_{i j}$. Let $L=D-A$ be the Laplacian matrix of $A$. The matrix $L$ is symmetric, positive-semidefinite. Its eigenvalues are non-negative reals and its smallest one is 0 with eigenvector $\mathbf{1}_{n}$, vector of all ones. We denote these eigenvalues in increasing order as

$$
0=\lambda_{1}(L) \leq \lambda_{2}(L) \leq \ldots \leq \lambda_{n}(L) .
$$

The second smallest eigenvalue of $L, \lambda_{2}(L)$ is usually referred to as the algebraic connectivity of the network. Let $\varepsilon>0$. Let $W=I-\varepsilon L$. The matrix $W$ is symmetric and therefore also has real eigenvalues. We denote them in decreasing order as follows:

$$
1=\lambda_{1}(W) \geq \lambda_{2}(W) \geq \ldots \geq \lambda_{n}(W),
$$

so that we have for $i \in \mathcal{N}$,

$$
\lambda_{i}(W)=1-\varepsilon \lambda_{i}(L) .
$$

Lemma 2 (Lemma 8 [KM07]). Let $J=\frac{1}{n} 1_{n} 1_{n}^{T}$. The spectral radius of $W-J$ is $\max \left(1-\varepsilon \lambda_{2}(L),-1+\varepsilon \lambda_{n}(L)\right)$. 
Remark 3. In the rest of the paper, we shall assume $\varepsilon<\frac{1}{\lambda_{n}(L)}$ for all Laplacian matrix considered. This is always possible by choosing $\varepsilon=\frac{1}{2(n-1)}$ (see e.g. [GR01]). Under this assumption, the spectral radius of $W-J$ is $1-\varepsilon \lambda_{2}(L)$. This assumption also yields $\varepsilon<\frac{1}{\lambda_{2}(L)}$ and thus $1-\varepsilon \lambda_{2}(L)>0$.

To a communication weight matrix $A$, we associate a weighted graph $G=(\mathcal{N}, \mathcal{E}, A)$ consisting of a set of nodes $\mathcal{N}=\{1, \ldots, n\}$ and a set of edges given by a relation $\mathcal{E} \subseteq \mathcal{N} \times \mathcal{N}$ such that $(i, j) \in \mathcal{E}$ iff $a_{i j}>0$. According to assumption $1, \mathcal{E}$ is symmetric $((i, j) \in \mathcal{E}$ iff $(j, i) \in \mathcal{E})$ and anti-reflexive $(\forall i \in \mathcal{N},(i, i) \notin \mathcal{E})$. If $(i, j) \in \mathcal{E}$, we say that $i$ is a neighbor of $j$. A path between $i$ and $j$ is a sequence of nodes $\left(i_{1}, i_{2}, \ldots, i_{p}\right)$ such that $i_{1}=i, i_{p}=j$ and $\forall k \in\{1, \ldots, p-1\},\left(i_{k}, i_{k+1}\right) \in \mathcal{E}$. We shall consider throughout this paper paths without loops, i.e. for all $k, k^{\prime} \in\{1, \ldots, p-1\}$ $k \neq k^{\prime}$ implies $i_{k} \neq i_{k^{\prime}}$. A graph is said to be connected if for every couple of nodes $(i, j) \in \mathcal{N} \times \mathcal{N}$ such that $i \neq j$, there exists a path between $i$ and $j$.

We define a partial order relation on weighted graphs.

Definition 4. A graph $G^{\prime}=\left(\mathcal{N}, \mathcal{E}^{\prime}, A^{\prime}\right)$ is said to be a (spanning) subgraph of $G$, which is denoted

$$
G^{\prime} \leqslant G
$$

when for all $i, j \in \mathcal{N}, A_{i j}^{\prime} \leq A_{i j}$.

The following proposition shows that $\lambda_{2}(L)$ is a good measure of the connectivity of the graph:

Lemma 5 ([GR01]). Let $L$ and $L^{\prime}$ be the Laplacians matrices associated to graphs $G$ and $G^{\prime}$ respectively.

- $G$ is connected iff $\lambda_{2}(L)>0$.

- If $G^{\prime} \leqslant G$, i.e. $G^{\prime}$ is a subgraph of $G$, then $\lambda_{2}\left(L^{\prime}\right) \leq \lambda_{2}(L)$.

\section{B. Consensus over Random Networks}

In this section, we apply the algebraic formalism to system (1) to derive a useful result. This approach was for instance used in [TSJ06] and [KM07].

Let $x(t)=\left(x_{1}(t)^{T}, \ldots, x_{n}(t)^{T}\right)^{T} \in \mathbb{R}^{\text {nd }}$ and $v(t)=$ $\left(v_{1}(t)^{T}, \ldots, v_{n}(t)^{T}\right)^{T} \in \mathbb{R}^{n d}$ be the stacked vectors of positions and velocities, respectively. We also define the stacked vectors of initial positions and velocities: $x^{0}=\left(x_{1}^{0^{T}}, \ldots, x_{n}^{0^{T}}\right)^{T} \in \mathbb{R}^{n d}$ and $v^{0}=\left(v_{1}^{0^{T}}, \ldots, v_{n}^{0^{T}}\right)^{T} \in \mathbb{R}^{n d}$. Let $\mathbf{L}(t)=L(t) \otimes I_{d}$, $\mathbf{W}(t)=W(t) \otimes I_{d}$ and $\mathbf{J}(t)=J(t) \otimes I_{d}$ where $I_{d}$ is the $d \times d$ identity matrix and $\otimes$ denotes the Kronecker product. Then, system (1) becomes in matrix form

$$
\begin{aligned}
& x(t+1)=x(t)+\varepsilon v(t) \\
& v(t+1)=\mathbf{W}(t) v(t)=\left(I_{n d}-\varepsilon \mathbf{L}(t)\right) v(t) .
\end{aligned}
$$

Since $L(t)$ is symmetric and $\mathbf{1}_{n}$ is an eigenvector associated to eigenvalue 0 , the average of the velocities $v^{*}=1 / n \cdot\left(\left(\mathbf{1}_{n} \otimes\right.\right.$ $\left.\left.I_{d}\right)^{T} v^{0}\right) \otimes \mathbf{1}_{n}$ is preserved by (4). It follows that if the agents achieve a flocking behavior, the common asymptotic velocity is necessarily $v^{*}$.

For $i \in \mathcal{N}$, let $\delta_{i}(t)=v_{i}(t)-v^{*}$, we define the velocity disagreement vector $\delta(t)=\left(\delta_{1}(t)^{T}, \ldots, \delta_{n}(t)^{T}\right)^{T}$. Let $y_{i}(t)=$ $x_{i}(t)-\varepsilon v^{*} t$ and $y(t)=\left(y_{1}(t)^{T}, \ldots, y_{n}(t)^{T}\right)^{T}$. Denote $z(t)=y(t)-$ $x^{0}$. We have $z(0)=0$. The vector $y(t)$ essentially gives the relative positions of the agents, as we have

$$
\forall i, j \in \mathcal{N}, x_{i}(t)-x_{j}(t)=y_{i}(t)-y_{j}(t)
$$

whereas $z(t)$ gives the modifications of the relative positions since

$$
\forall i, j \in \mathcal{N}, z_{i}(t)-z_{j}(t)=x_{i}(t)-x_{j}(t)-\left(x_{i}^{0}-x_{j}^{0}\right) .
$$

The dynamics of $z$ and $\delta$ are as follows:

$$
\begin{aligned}
& z(t+1)=z(t)+\varepsilon \delta(t), \\
& \delta(t+1)=\mathbf{W}(t) \delta(t) .
\end{aligned}
$$

Lemma 6 (Lemma 7 [KM07]). For all time $t \in \mathbb{N}$,

$$
\|\delta(t+1)\| \leq\left(1-\varepsilon \lambda_{2}(L(t))\right)\|\delta(t)\| .
$$

Lemma 6 gives a sufficient condition for flocking, i.e. $\prod_{t=0}^{\infty}\left(1-\varepsilon \lambda_{2}(L(t))\right)=0$. However, this condition is not simple to verify since $L(t)$ is a position dependent matrix. This is why, in the next section, we introduce a bounding function of $\|\delta(t)\|$ which is easier to deal with.

\section{SUFFICIENT CONDITION FOR FLOCKING}

\section{A. Main result}

In this section, we propose a theorem giving sufficient conditions for flocking. To do so, we introduce the variable $\rho>0$ which represents the maximal perturbation allowed on the relative positions of agents. We then define the weighted graph $\tilde{G}(t)=(\mathcal{N}, \tilde{\mathcal{E}}(t), \tilde{A}(t))$ corresponding to the initial relative distance between agents perturbed by $\rho$, where $\tilde{A}(t)=\left(\tilde{a}_{i j}(t)\right)$ with

$$
\tilde{a}_{i j}(t)=a_{i j}\left(t,\left\|x_{i}(0)-x_{j}(0)\right\|+\rho\right),
$$

where the functions $a_{i j}$ are the interaction weights used in system (1). We denote $\tilde{L}(t)$ the Laplacian matrix of weighted graph $\tilde{G}(t)$. Notice that the graph $\tilde{G}(t)$ is not explicitly statedependant anymore.

These notation allow us to state the main theoretical theorem of the paper :

Theorem 7. Flocking is achieved if ${ }^{2}$

$$
\left(\sum_{t=0}^{+\infty} \prod_{w=0}^{t-1}\left(1-\varepsilon \lambda_{2}(\tilde{L}(w))\right)\right)\|\delta(0)\| \leq \frac{\rho}{\varepsilon \sqrt{2}} .
$$

As given the computation of the sufficient condition for flocking given in Theorem 7 may not seem obvious. We shall see how this can be done for specific communication rules in section $\mathrm{V}$. We can already qualitatively comment on this condition. The condition depends on the initial velocities through the vector $\|\delta(0)\|$. As expected, flocking is more likely to occur if the initial velocity disagreement is small. The dependency on the initial positions comes from $\lambda_{2}(\tilde{L}(w))$. Notice that according to assumption 1 and lemma $5, \lambda_{2}(\tilde{L}(w))$ decreases when the distance between agents increases, making flocking less likely to occur. The robustness $\rho$ appears twice in

\footnotetext{
${ }^{2}$ In the equation and throughout the paper, we use convention $\prod_{w=0}^{-1} f_{w}=$ $\prod_{w \in \emptyset} f_{w}=1$, for any $f_{w} \in \mathbb{R}$, and $\sum_{\tau=0}^{-1} g_{\tau}=\sum_{\tau \in \emptyset} g_{\tau}=0$, for any $g_{\tau} \in \mathbb{R}$.
} 
the condition since $\lambda_{2}(\tilde{L}(w))$ also depends on it. The optimal choice of parameter $\rho$ will be discussed in section VI-A.

The proof of the previous theorem requires intermediate results which we derive in the next two sections. We thus postpone the proof to the end of section IV-C. Essentially, we will show that for all time $t \geq 0$,

$$
\tilde{G}(t) \preccurlyeq G(t),
$$

i.e. $\tilde{G}(t)$ bounds the minimal quantity of interactions taking place in $G(t)$. To do so, we combine the following steps:

- equation (8) imposes a minimal convergence rate for the velocity disagreement, which in turns gives a bound on the distance modification at time $t+1$ (see lemma 9).

- if the distances between agents $\left\|x_{i}(t+1)-x_{j}(t+1)\right\|$ are not modified more than the robustness $\rho$ then equation (8) is satisfied at time $t+1$ (see proposition 8).

An induction on time $t$ allows to conclude. The fact that equation 8 implies flocking lies implicitly in the fact that it bounds the distances between agents and thus forces the velocities to converge (see Theorem 10).

\section{B. Robustness of the interaction graph}

The definition of graph $\tilde{G}(t)$ and assumption 1 yields a sufficient condition for preserving the graph $\tilde{G}(t)$ in $G(t)$ over time :

Proposition 8. Let $t \in \mathbb{N}$. Let $z(t)=y(t)-x^{0}$ as in section III-B. If

$$
\|z(t)\| \leq \frac{\rho}{\sqrt{2}},
$$

then $\tilde{G}(t)$ is a subgraph of $G(t)$, i.e. $\tilde{G}(t) \leqslant G(t)$, where the partial order $\leqslant i$ s defined in definition 4.

Proof. Let $i, j \in \mathcal{N}$ distinct. We have

$$
-2\left\langle z_{i}(t) \mid z_{j}(t)\right\rangle \leq\left\|z_{i}(t)\right\|^{2}+\left\|z_{j}(t)\right\|^{2} .
$$

Thus,

$$
\begin{aligned}
\left\|z_{i}(t)-z_{j}(t)\right\|^{2} & =\left\|z_{i}(t)\right\|^{2}+\left\|z_{j}(t)\right\|^{2}-2\left\langle z_{i}(t) \mid z_{j}(t)\right\rangle \\
& \leq 2\left(\left\|z_{i}(t)\right\|^{2}+\left\|z_{j}(t)\right\|^{2}\right) \\
& \leq 2\|z(t)\|^{2}
\end{aligned}
$$

Then, using the assumption of the proposition, we have $\| z_{i}(t)-$ $z_{j}(t) \| \leq \rho$, so that equation (5) gives

$$
\begin{aligned}
& \left\|x_{i}(t)-x_{j}(t)\right\|=\left\|x_{i}(t)-x_{j}(t)-\left(x_{i}^{0}-x_{j}^{0}\right)+\left(x_{i}^{0}-x_{j}^{0}\right)\right\| \\
& \leq\left\|x_{i}(t)-x_{j}(t)-\left(x_{i}^{0}-x_{j}^{0}\right)\right\|+\left\|x_{i}^{0}-x_{j}^{0}\right\| \\
& \leq\left\|z_{i}(t)-z_{j}(t)\right\|+\left\|x_{i}^{0}-x_{j}^{0}\right\| \\
& \leq \rho+\left\|x_{i}^{0}-x_{j}^{0}\right\| .
\end{aligned}
$$

This, along with assumption 1, allows to conclude.

We will show in the application section that under certain conditions, the assumption required in proposition 8 is verified, i.e. the modification on the distances between agents is always smaller than maximal perturbation $\rho$ (which explains its name).

In the next section, we explain how to use proposition 8 to derive a sufficient condition for flocking.

\section{Bounding processes}

We define a system of two variables lying in $\mathbb{R}^{+},(p, q)$ which will serve to bound system (1) :

$$
\begin{aligned}
& p(t+1)=p(t)+\varepsilon q(t), \\
& q(t+1)=\left(1-\varepsilon \lambda_{2}(\tilde{L}(t))\right) q(t),
\end{aligned}
$$

where $p(0)=0$ and $q(0)=\|\delta(0)\|$. Under a certain assumption, we shall show that $p$ bounds $\|z\|$, the distance modification between agents while $q$ serves as a bound to the velocity disagreement vector $\|\delta\|$ (see equation (6)). As we shall see in the application, the behavior of $(p, q)$ is easier to characterize than the one of $(z, \delta)$.

We can now state the bounding property:

Lemma 9. We have

$$
\left(\forall t \in \mathbb{N}, p(t)<\frac{\rho}{\sqrt{2}}\right) \Rightarrow\left(\forall t \in \mathbb{N},\left\{\begin{array}{l}
\lambda_{2}(L(t)) \geq \lambda_{2}(\tilde{L}(t)) \\
\|\delta(t)\| \leq q(t) \\
\|z(t)\| \leq p(t)
\end{array}\right) .\right.
$$

Proof. We show the result by induction on time. Assume that the left-hand side of the implication is satisfied. By definition, $q(0)=\|\delta(0)\|$ and $p(0)=\|z(0)\|=0$. Assume the result is true for some time $t:\|\delta(t)\| \leq q(t)$ and $\|z(t)\| \leq p(t)$. The inequality on the positions at time $t+1$ comes as follows:

$$
\begin{aligned}
\|z(t+1)\| & =\|z(t)+\varepsilon \delta(t)\| \leq\|z(t)\|+\varepsilon\|\delta(t)\| \\
& \leq p(t)+\varepsilon q(t)=p(t+1) .
\end{aligned}
$$

For the inequality on the velocities, we have $\|z(t)\| \leq p(t)<$ $\rho / \sqrt{2}$, where we used the left-hand side of the implication. Thus, we can apply proposition 8 along with Lemma 5 to obtain $\lambda_{2}(L(t)) \geq \lambda_{2}(\tilde{L}(t))$. Then, Lemma 6 gives

$$
\begin{aligned}
\|\delta(t+1)\| & \leq\left(1-\varepsilon \lambda_{2}(L(t))\right)\|\delta(t)\| \\
& \leq\left(1-\varepsilon \lambda_{2}(\tilde{L}(t))\right)\|\delta(t)\| \\
& \leq\left(1-\varepsilon \lambda_{2}(\tilde{L}(t))\right) q(t)=q(t+1) .
\end{aligned}
$$

This last lemma leads to the following result:

Theorem 10. If for all time $t, p(t) \leq \frac{\rho}{\sqrt{2}}$ then flocking is achieved, i.e. $\lim _{t \rightarrow+\infty}\|\delta(t)\|=0$.

Proof. We have that $\lambda_{2}(\tilde{L}(t))>0$ (see algebraic graph theory section III-A). Also, since $\lambda_{2}(\tilde{L}(t))<\lambda_{2}(L(t))$ (see Lemma 9). These two facts along with Remark 3 give that $\left(1-\varepsilon \lambda_{2}(\tilde{L}(t))\right) \in$ $(0,1)$. Thus, since sequence $q$ is defined by system $(9)$, it is non-negative and non-increasing. As a consequence, sequence $q$ must converge. It is easy to see that if $\lim _{t \rightarrow \infty} q(t)>0$ then $p$ diverges. Thus, $\lim _{t \rightarrow \infty} q(t)=0$. Using Lemma 9 , we have $\|\delta(t)\| \leq$ $q(t)$, thus $\lim _{t \rightarrow \infty}\|\delta(t)\|=0$ which guarantees flocking.

In order to use the previous Lemma, we need to be able to characterize the evolution of $p$ in function of the initial configurations of the system. This will be done in the application section.

Proof of Theorem 7. Notice that, from system (9), for $t \in \mathbb{N}$,

$$
p(t)=\varepsilon\left(\sum_{\tau=0}^{t-1} \prod_{u=0}^{\tau-1}\left(1-\varepsilon \lambda_{2}(\tilde{L}(u))\right)\right)\|\delta(0)\|,
$$


which, using Remark 3, shows that $p$ is a non-decreasing sequence. Thus, Theorem 7 becomes a direct corollary of Theorem 10 .

\section{Applications}

We now present two applications to our main theoretical result. The first one assume that agents interact when at a distance smaller than a fixed interaction radius $R$, whereas in the second one the interaction radiuses are allowed to differ with the agents and are subject to random variations over time.

\section{A. Interactions under fixed communication radius}

We consider $n$ agents evolving according to system (1). We suppose that two agents $i$ and $j \in \mathcal{N}$ interact when their distance is smaller than a certain interaction radius $R>0$, i.e.

$$
a_{i j}\left(t,\left\|x_{i}(t)-x_{j}(t)\right\|\right)=\chi\left(\left\|x_{i}(t)-x_{j}(t)\right\| \leq R\right)
$$

where the $\chi()$ is the characteristic function (for an assertion $A, \chi(A)=1$ if $A$ is true and 0 otherwise). Such interactions weights satisfy assumption 1 . Therefore, Theorem 7 applies : flocking is achieved if

$$
\left(\sum_{t=0}^{+\infty} \prod_{w=0}^{t-1}\left(1-\varepsilon \lambda_{2}(\tilde{L}(w))\right)\right)\|\delta(0)\| \leq \frac{\rho}{\varepsilon \sqrt{2}},
$$

where $\tilde{L}(w)$ is the Laplacian matrix associated to adjacency matrix $\tilde{A}=\left(\tilde{a}_{i j}(t)\right)_{i j}$ defined so that, according to equation (7),

$$
\tilde{a}_{i j}(t)=\chi\left(\left\|x_{i}^{0}-x_{j}^{0}\right\|+\rho \leq R\right) .
$$

The matrix $\tilde{L}$ is thus independent of time. A consequence of this fact is the following theorem:

Theorem 11. Flocking is achieved if

$$
\|\delta(0)\| \leq \frac{\lambda_{2}(\tilde{L}(0)) \rho}{\sqrt{2}} .
$$

Proof. Using the fact that $\tilde{L}$ is independent of time, we can rewrite the assumption in Theorem 7 as

$$
\begin{aligned}
\left(\sum_{t=0}^{+\infty}\left(1-\varepsilon \lambda_{2}(\tilde{L}(0))\right)^{t}\right)\|\delta(0)\| \leq \frac{\rho}{\varepsilon \sqrt{2}} & \Leftrightarrow \frac{\|\delta(0)\|}{\varepsilon \lambda_{2}(\tilde{L}(0))} \leq \frac{\rho}{\varepsilon \sqrt{2}} \\
& \Leftrightarrow\|\delta(0)\| \leq \frac{\lambda_{2}(\tilde{L}(0)) \rho}{\sqrt{2}},
\end{aligned}
$$

where for the first equivalence, we have computed the infinite sum of a geometric sequence, since according to Remark 3 and Lemma $9,1-\varepsilon \lambda_{2}(\tilde{L}(0)) \in(0,1)$. The last equation is precisely the assumption required in Theorem 11. Theorem 7 allows to conclude.

\section{B. Interactions under random communication radius}

In this section, we focus on communication of agents defined by a metric rule parametrized by random interaction radiuses. To do so, we assume that each agent $i$ has a sensing radius $R_{i}(t)$ following uniform distribution over the interval $[\bar{R}-\hat{R} / 2, \bar{R}+\hat{R} / 2], \bar{R}>0$ being the average communication range and $\hat{R} \in[0,2 \bar{R}]$ the width of the possible variations ${ }^{3}$. The communication weights are then defined by

$$
a_{i j}(t)=\chi\left(\left\|x_{i}(t)-x_{j}(t)\right\| \leq R_{i j}(t) \text { and } i \neq j\right),
$$

where

$$
R_{i j}(t)=\min \left(R_{i}(t), R_{j}(t)\right) .
$$

Taking $R_{i j}(t)=\min \left(R_{i}(t), R_{j}(t)\right)$ means that two agents interact if and only if both agents can sense the other. Thus if the sensing is low range, so will be the communication. It has been shown in [BW09] that the uniform distribution is a good approximation of the log-normal shadowing statistical model for wireless networks and is, as such, of practical relevance.

Such metric interactions make the communication weights symmetric and, given a fixed radius $R_{i j}(t)$, decreasing with the distance between agents and thus verify assumption 1 . Therefore, Theorem 7 applies. Moreover, the sensing radiuses $R_{i}$ are independent and identically distributed in time (i.i.d.). Since $R_{i j}$ is the minimum of two time-independent random variables, it is itself time-independent. In the first part of the section, we shall see how these facts helps to determine precise a lower bound on the probability of flocking.

1) Probability of flocking: In this section we make use of notation $\tilde{G}(t), \tilde{A}(t)=\left[\tilde{a}_{i j}(t)\right]$, the adjacency matrix, and $\tilde{L}(t)$ the Laplacian associated to matrix $\tilde{A}(t)$. Since $R_{i j}(t)$ are time-independent processes, so are $\tilde{a}_{i j}(t), \tilde{A}(t)$ and $\tilde{L}(t)$. Thus, $\mathbb{E}\left(\lambda_{2}(\tilde{L}(t))\right)$ is independent of time. Then, let $\tilde{\lambda}_{2}=\mathbb{E}\left(\lambda_{2}(\tilde{L}(t))\right)$. In the rest of the section, we will assume $\tilde{\lambda}_{2}>0$. This occurs iff $\tilde{G}(t)$ is connected with non-zero probability (this is true if for instance $\rho=0$ provided that $G(0)$ is connected with non-zero probability).

According to [TSJ06], if the communication graph is connected on average for all time (i.e. $\forall t \geq 0, \mathbb{E}\left(\lambda_{2}(L(t))\right)>0$ ), then velocity alignment is guaranteed. Lemma 5 states that if $\tilde{G}(t) \subseteq G(t), \mathbb{E}\left(\lambda_{2}(L(t))\right) \geq \mathbb{E}\left(\lambda_{2}(\tilde{L}(t))\right)>0$. Consequently, the preservation of $\tilde{G}(t)$ in the communication graph over time allows to characterize the velocity alignment rate in function of $\mathbb{E}\left(\lambda_{2}(\tilde{L}(t))\right)$. This discussion leads to the following theorem:

Theorem 12. Assume that $\rho>0$ and $\tilde{\lambda}_{2}>0$. Moreover suppose $\|\delta(0)\| \leq \frac{\rho \tilde{\lambda}_{2}}{\sqrt{2}}$. Then the probability that flocking is achieved is higher than

$$
1-\frac{1}{1+\kappa} \text { where } \kappa=\frac{1}{v_{\varepsilon}}\left(\frac{\rho \tilde{\lambda}_{2}}{\sqrt{2}\|\delta(0)\|}-1\right)^{2},
$$

and $v_{\varepsilon}=\frac{\varepsilon \operatorname{var}\left(\lambda_{2}(\tilde{L}(t))\right)}{\left(2 \tilde{\lambda}_{2}-\varepsilon \mathbb{E}\left(\lambda_{2}(\tilde{L}(t))^{2}\right)\right)} \in[0,1]$, where $\operatorname{var}\left(\lambda_{2}(\tilde{L}(t))\right)=$ $\mathbb{E}\left(\left(\lambda_{2}(\tilde{L}(t))\right)^{2}\right)-\mathbb{E}\left(\lambda_{2}(\tilde{L}(t))\right)^{2}$ is the variance of $\lambda_{2}(\tilde{L}(t))$.

Remark 13. As given, the result allows us to obtain the probability of flocking arbitrarily close to 1 in three ways :

- First, one can choose the initial disagreement vector sufficiently small.

- Second, since $\lim _{\varepsilon \rightarrow 0} v_{\varepsilon}=0$, assume the condition on $\|\delta(0)\|$ is satisfied, and then choose $\varepsilon$ sufficiently small.

- Finally, the variance $\operatorname{var}\left(\lambda_{2}(\tilde{L}(t))\right)$ converges to 0 when $\hat{R}$ vanishes. In this case, the limit bound is exactly the one

\footnotetext{
${ }^{3}$ Rigorously, we should write $R_{i}(t, \omega)$ where $\omega \in \Omega$, the set of probabilistic events. For simplicity of notation, we drop $\omega$, unless necessary.
} 
given by Theorem 11, for the deterministic setting. This is consistent since when $\hat{R}$ vanishes, the stochasticity of the system disappears.

Notice that, on the opposite, choosing $\rho$ arbitrarily high would not work since $\tilde{\lambda}_{2}$ is a decreasing function of $\rho$. We detail the optimal choice of $\rho$ in section VI-A.

Remark 14. The result remains valid for any communication rule leading to an independent and identically distributed symmetric communication graph $G(t)$.

The proof of the theorem requires characterizing the evolution of the bound $p$ on the modification of the distances in function of the initial configuration of the system, and is therefore relocated at the end of the next section.

Lemma 15. For all $t \in \mathbb{N}$, we have

$$
\left\{\begin{array}{l}
\mathbb{E}(q(t+1))=\alpha \mathbb{E}(q(t)) \\
\mathbb{E}\left(q(t+1)^{2}\right)=\beta \mathbb{E}\left(q(t)^{2}\right)
\end{array}\right.
$$

where

$$
\left\{\begin{aligned}
\alpha & =\mathbb{E}\left(1-\varepsilon \lambda_{2}(\tilde{L}(t))\right)=1-\varepsilon \tilde{\lambda}_{2} \\
\beta & =\mathbb{E}\left(\left(1-\varepsilon \lambda_{2}(\tilde{L}(t))\right)^{2}\right) \\
& =1-2 \varepsilon \tilde{\lambda}_{2}+\varepsilon^{2} \mathbb{E}\left(\lambda_{2}(\tilde{L}(t))^{2}\right)
\end{aligned}\right.
$$

Proof. The Lemma can be derived easily using the fact that $\tilde{L}(t)$ is independent of $q(t)$.

The bounding process is useful only if the variance of $q(t)$ converges to 0 . This is the case under the condition assumed in Remark 3 regarding $\varepsilon$ :

Remark 16. Assuming $\varepsilon<1 / \lambda_{n}(\tilde{L}(t))$ for all t as in Remark 3 , both $\alpha$ and $\beta$ lie in interval $(0,1)$.

The previous remark holds since, according to Remark 3, $1-\varepsilon \lambda_{2}(\tilde{L}(t)) \in(0,1)$ for any realization of the random radiuses $R_{i}(t)$. Then, the definition of $\alpha$ and $\beta$ allows us to conclude.

A direct corollary of Lemma 15 is the following :

Corollary 17. For all $t, u \in \mathbb{N}$, we have

$$
\left\{\begin{array}{l}
\mathbb{E}(q(t))=\alpha^{t} q(0), \\
\mathbb{E}\left(q(t)^{2}\right)=\beta^{t} q(0)^{2}, \\
\mathbb{E}(q(t+u) q(t))=\alpha^{u} \mathbb{E}\left(q(t)^{2}\right)
\end{array}\right.
$$

Proof. The first and second equations are straight-forward. The third one is also a consequence of $\tilde{L}(t)$ being i.i.d.:

$$
\begin{aligned}
\mathbb{E}(q(t+u) q(t)) & =\mathbb{E}\left(\prod_{j=0}^{u-1}\left(1-\varepsilon \lambda_{2}(\tilde{L}(t+j))\right) q(t)^{2}\right) \\
& =\left(1-\varepsilon \mathbb{E}\left(\lambda_{2}(\tilde{L}(t))\right)^{u} \mathbb{E}\left(q(t)^{2}\right) .\right.
\end{aligned}
$$

Noticing that $p(t)=\varepsilon \sum_{k=0}^{t-1} q(k)$ and with the assumption $\tilde{\lambda}_{2}>$ 0 , the following also holds :

Lemma 18. If we assume $\tilde{\lambda}_{2}>0$, we have, for all $t \in \mathbb{N}$,

$$
\left\{\begin{array}{l}
\mathbb{E}(p(t))=\varepsilon \frac{q(0)}{1-\alpha}+o(t), \\
\sigma^{2}(p(t))=\varepsilon^{2} \frac{\left(\beta-\alpha^{2}\right) q(0)^{2}}{(1-\alpha)^{2}(1-\beta)}+o(t)
\end{array}\right.
$$

where $\sigma^{2}(p(t))=\mathbb{E}\left(p(t)^{2}\right)-\mathbb{E}(p(t))^{2}$ and $o(t)$ is a function converging to 0 when time $t$ approaches to infinity.

Proof. The first equality comes from the above remark and the first equation in Corollary 17:

$$
\begin{aligned}
\mathbb{E}(p(t)) & =\varepsilon \sum_{k=0}^{t-1} \mathbb{E}(q(k))=\varepsilon \sum_{k=0}^{t-1} \alpha^{k} q(0) \\
& =\varepsilon \frac{1-\alpha^{t}}{1-\alpha} q(0)=\varepsilon \frac{q(0)}{1-\alpha}+o(t)
\end{aligned}
$$

where we used $|\alpha|<1$ as given in Remark 16 .

For the second equality,

$$
\begin{aligned}
p(t)^{2} & =\varepsilon^{2} \sum_{k, j=0}^{t-1} q(k) q(j) \\
& =\varepsilon^{2}\left(\sum_{k=0}^{t-1} q(k)^{2}+2 \sum_{k<j} q(j) q(k)\right) \\
& =\varepsilon^{2} \sum_{k=0}^{t-1}\left(q(k)^{2}+2 \sum_{u=1}^{t-1-k} q(k+u) q(k)\right) .
\end{aligned}
$$

Using the linearity of the expectation, Corollary 17 and $|\alpha|<1$, we obtain

$$
\begin{aligned}
\mathbb{E}\left(p(t)^{2}\right) & =\varepsilon^{2} \sum_{k=0}^{t-1}\left(\mathbb{E}\left(q(k)^{2}\right)+2 \sum_{u=1}^{t-1-k} \alpha^{u} \mathbb{E}\left(q(k)^{2}\right)\right) \\
& =\varepsilon^{2} \sum_{k=0}^{t-1}\left(1+2\left(\frac{1-\alpha^{t-k}}{1-\alpha}-1\right)\right) \mathbb{E}\left(q(k)^{2}\right) \\
& =\varepsilon^{2} \sum_{k=0}^{t-1}\left(\frac{1+\alpha}{1-\alpha}-\left(\frac{2 \alpha^{t}}{1-\alpha} \alpha^{-k}\right)\right) \beta^{k} q(0)^{2} \\
& =\varepsilon^{2}\left(\frac{1+\alpha}{1-\alpha} \sum_{k=0}^{t-1} \beta^{k}-\frac{2 \alpha^{t}}{1-\alpha} \sum_{k=0}^{t-1}\left(\frac{\beta}{\alpha}\right)^{k}\right) q(0)^{2} .
\end{aligned}
$$

Moreover, Remark 3 gives $0<\varepsilon<\frac{1}{\lambda_{2}(\tilde{L}(t))}$ for any realization of the random radiuses $R_{i}(t)$ and choice $r_{i j}$. Thus,

$$
\varepsilon \mathbb{E}\left(\lambda_{2}(\tilde{L}(t))^{2}\right)=\mathbb{E}\left(\varepsilon \lambda_{2}(\tilde{L}(t))^{2}\right)<\mathbb{E}\left(\lambda_{2}(\tilde{L}(t))\right)=\tilde{\lambda}_{2} .
$$

So, we have

$$
\beta=1-2 \varepsilon \tilde{\lambda}_{2}+\varepsilon^{2} \mathbb{E}\left(\lambda_{2}(\tilde{L}(t))^{2}\right)<1-2 \varepsilon \tilde{\lambda}_{2}+\varepsilon \tilde{\lambda}_{2}=\alpha .
$$

Thus, $\beta \neq \alpha$. Also $|\beta|<1$, so we have

$$
\begin{aligned}
& \mathbb{E}\left(p(t)^{2}\right)=\varepsilon^{2}\left(\frac{1+\alpha}{1-\alpha} \frac{1-\beta^{t}}{1-\beta}-\frac{2 \alpha^{t}}{1-\alpha} \frac{1-(\beta / \alpha)^{t}}{1-(\beta / \alpha)}\right) q(0)^{2} \\
& =\varepsilon^{2}\left(\frac{1+\alpha}{1-\alpha} \frac{1-\beta^{t}}{1-\beta}-\frac{2}{1-\alpha} \frac{\alpha^{t}-\beta^{t}}{1-(\beta / \alpha)}\right) q(0)^{2} \\
& =\varepsilon^{2} \frac{1+\alpha}{(1-\alpha)(1-\beta)} q(0)^{2}+o(t) .
\end{aligned}
$$

Then,

$$
\begin{aligned}
& \sigma^{2}(p(t))=\mathbb{E}\left(p(t)^{2}\right)-\mathbb{E}(p(t))^{2} \\
& =\varepsilon^{2}\left(\frac{1+\alpha}{(1-\alpha)(1-\beta)}-\frac{1}{(1-\alpha)^{2}}\right) q(0)^{2}+o(t) .
\end{aligned}
$$

Reformulating the last right-hand side leads to the expected result. 
Finally, we translate the equation from the previous lemma in terms of the expectation and deviation of the algebraic connectivity:

Corollary 19. For all $t \in \mathbb{N}$, we have

$$
\left\{\begin{array}{l}
\mathbb{E}(p(t))=\frac{\|\delta(0)\|}{\tilde{\lambda}_{2}}+o(t), \\
\sigma^{2}(p(t))=\frac{v_{\varepsilon}\|\delta(0)\|^{2}}{\tilde{\lambda}_{2}^{2}}+o(t),
\end{array}\right.
$$

where $v_{\varepsilon} \in[0,1]$ is defined in Theorem 12 .

Remark 20. Since $\lim _{\varepsilon \rightarrow 0} v_{\varepsilon}=0$, the previous result shows that one can obtain the limit variance of $p(t)$ as small as desired by choosing $\varepsilon$ small enough.

Proof. Notice that the definitions of $\alpha$ and $\beta$ give

$$
\begin{aligned}
& 1-\alpha=\varepsilon \tilde{\lambda}_{2}, 1-\beta=\varepsilon\left(2 \tilde{\lambda}_{2}-\varepsilon \mathbb{E}\left(\lambda_{2}(\tilde{L}(t))^{2}\right)\right), \\
& 1+\alpha=2-\varepsilon \tilde{\lambda}_{2}, \beta-\alpha^{2}=\varepsilon^{2}\left(\mathbb{E}\left(\lambda_{2}(\tilde{L}(t))^{2}\right)-\tilde{\lambda}_{2}^{2}\right) .
\end{aligned}
$$

Then, the first equation is straightforward. The second is derived as follows:

$$
\begin{aligned}
\sigma^{2}(p(t)) & =\varepsilon^{2} \frac{\left(\beta-\alpha^{2}\right) q(0)^{2}}{(1-\alpha)^{2}(1-\beta)}+o(t) \\
& =\varepsilon^{4} \frac{\left(\mathbb{E}\left(\lambda_{2}(\tilde{L}(t))^{2}\right)-\tilde{\lambda}_{2}^{2}\right)\|\delta(0)\|^{2}}{\varepsilon^{3} \tilde{\lambda}_{2}^{2}\left(2 \tilde{\lambda}_{2}-\varepsilon \mathbb{E}\left(\lambda_{2}(\tilde{L}(t))^{2}\right)\right)}+o(t) \\
& =\frac{v_{\varepsilon}\|\delta(0)\|^{2}}{\tilde{\lambda}_{2}^{2}}+o(t) .
\end{aligned}
$$

The fact that $v_{\varepsilon} \geq 0$ can be deduced from $\sigma^{2}(p(t) \geq 0$. The inequality $v_{\varepsilon} \leq 1$ comes from $\varepsilon \mathbb{E}\left(\lambda_{2}(\tilde{L}(t))^{2}\right)<\tilde{\lambda}_{2}$ (see proof of Lemma 18), which gives $\tilde{\lambda}_{2}<2 \tilde{\lambda}_{2}-\varepsilon \mathbb{E}\left(\lambda_{2}(\tilde{L}(t))^{2}\right)$ and

$$
v_{\varepsilon}<\varepsilon \frac{\left(\mathbb{E}\left(\lambda_{2}(\tilde{L}(t))^{2}\right)-\tilde{\lambda}_{2}^{2}\right)}{\tilde{\lambda}_{2}} \leq \varepsilon \frac{\mathbb{E}\left(\lambda_{2}(\tilde{L}(t))^{2}\right)}{\tilde{\lambda}_{2}}<1 .
$$

We can now give the proof of the central theorem of this section.

Proof of Theorem 12. According to Theorem 10, the probability of flocking is higher than the probability of the event : $p(t) \leq \frac{\rho}{\sqrt{2}}$ for all time $t \in \mathbb{N}$. Denote $\mathcal{A}_{t}=\left\{\omega \in \Omega \mid p(t) \leq \frac{\rho}{\sqrt{2}}\right\}$ where $\Omega$ is the set of probabilistic events. Since $p$ is increasing, $\left(\mathcal{A}_{t}\right)$ is a decreasing sequence of events. Thus, we have

$$
\begin{aligned}
& \mathbb{P}\left(\forall t, p(t) \leq \frac{\rho}{\sqrt{2}}\right)=\mathbb{P}\left(\cap_{t \geq 0} \mathcal{A}_{t}\right) \\
& =\lim _{t \rightarrow+\infty} \mathbb{P}\left(\mathcal{A}_{t}\right)=\lim _{t \rightarrow+\infty} \mathbb{P}\left(p(t) \leq \frac{\rho}{\sqrt{2}}\right) \\
& =1-\lim _{t \rightarrow+\infty} \mathbb{P}\left(p(t)>\frac{\rho}{\sqrt{2}}\right) .
\end{aligned}
$$

The bound on the probability of flocking comes from the onesided Chebyshev's inequality:

$$
\mathbb{P}\left(p(t)>\frac{\rho}{\sqrt{2}}\right) \leq \frac{1}{1+k(t)}
$$

where $k(t)=\frac{\left(\frac{\rho}{\sqrt{2}}-\mathbb{E}(p(t))\right)^{2}}{\sigma^{2}(p(t))}$. We are only interested in the limit when $t$ diverges to infinity. We use Corollary 19 to derive a lower bound on the limit of $k(t)$ :

$$
\frac{\left(\frac{\rho}{\sqrt{2}}-\lim _{t \rightarrow \infty} \mathbb{E}(p(t))\right)^{2}}{\lim _{t \rightarrow \infty} \sigma^{2}(p(t))} \geq \frac{1}{v_{\varepsilon}}\left(\frac{\rho \tilde{\lambda}_{2}}{\sqrt{2}\|\delta(0)\|}-1\right)^{2} .
$$

\section{NUMERICAL VALIDATION}

In this section we show that our theoretical result can be implemented. As mentioned in remark 13, the system where agents interact within a fixed communication radius (see section V-A) is a particular case of the stochastic setting when the diameter of possible radiuses $\hat{R}$ is reduced to 0 . Thus, we only deal with the latter setting in the present section.

\section{A. Computation and optimization of the bound}

Theorem 12 gives a lower bound on the probability that a trajectory of system (1) reaches velocity alignment. To be useful, this bound should be computable. In this section, we explicit the quantities appearing in the bound which are not direct to compute: $\mathbb{E}\left(\lambda_{2}(\tilde{L}(t))\right)$ and $\mathbb{E}\left(\left(\lambda_{2}(\tilde{L}(t))\right)^{2}\right)$. Since the radiuses $R_{i}(t)$ for $i \in \mathcal{N}$ are i.i.d., these two quantities are independent of time. Thus, we drop the time dependency notation. The computation method will be the same for both $\mathbb{E}\left(\lambda_{2}(\tilde{L}(t))\right)$ and $\mathbb{E}\left(\left(\lambda_{2}(\tilde{L}(t))\right)^{2}\right)$, for this reason, we consider a function $\phi: \mathcal{M}_{n}(\mathbb{R}) \longrightarrow \mathbb{R}$ such that $\phi(\tilde{L})$ is summable.

We assume that $\rho \in[0, \bar{R}+\hat{R} / 2]$ is fixed unless otherwise mentioned. Denote $R_{\max }=\bar{R}+\hat{R} / 2$ and $R_{\min }=\bar{R}-\hat{R} / 2$. Denote, for $(j, i) \in \mathcal{N}, r_{i j}=\left\|x_{i}^{0}-x_{i}^{0}\right\|+\rho$. Also denote, for $i \in \mathcal{N}$, the set of uncertain neighbors of $i$ as

$$
\mathcal{U}_{i}=\left\{j \in \mathcal{N} \mid R_{\min } \leq r_{i j} \leq R_{\max }\right\} .
$$

Denote $d_{i}$ the cardinal of set $\mathcal{U}_{i}$. For a given $i$, we sort the $d_{i}$ values in $\mathcal{U}_{i}$ in increasing order and we denote $\left(r_{i}^{1}, r_{i}^{2}, \ldots, r_{i}^{d_{i}}\right)$ the resulting sequence.

Denote $r_{i}^{0}=R_{\min }$ and $r_{i}^{d_{i}+1}=R_{\max }$. These notation allow to state that $\tilde{L}$ is a piecewise constant function of $R_{i}$ for $i \in \mathcal{N}$ :

Lemma 21. Let $k_{i} \in\left\{0, \ldots, d_{i}\right\}$ for $i \in \mathcal{N}$. Then, for a fixed $\rho$, $\tilde{L}$ is constant when radiuses $R_{i}$ vary in $\left[r_{i}^{k_{i}}, r_{i}^{k_{i}+1}\right.$ [. Precisely, $\tilde{L}$ is given by

$$
\tilde{a}_{i j}=\chi\left(r_{i j} \leq \min \left(r_{i}^{k_{i}}, r_{j}^{k_{j}}\right)\right) .
$$

We denote $\tilde{L}\left(k_{1}, k_{2}, \ldots, k_{n}\right)$ this constant.

Proof of lemma 21. Assume that $k_{i} \in\left\{0, \ldots, d_{i}\right\}$ for $i \in \mathcal{N}$ fixed. Let $R_{i} \in\left[r_{i}^{k_{i}}, r_{i}^{k_{i}+1}\right.$ [ for $i \in \mathcal{N}$. Let $i, j \in \mathcal{N}$ distinct. We show that $\tilde{a}_{i j}$ is independent of the $R_{i}$. We have

$$
\begin{aligned}
\tilde{a}_{i j} & =\chi\left(\left\|x_{i}^{0}-x_{j}^{0}\right\|+\rho \leq \min \left(R_{i}, R_{j}\right)\right) \\
& =\chi\left(r_{i j} \leq \min \left(R_{i}, R_{j}\right)\right) .
\end{aligned}
$$

If $r_{i j}>R_{\max }, \tilde{a}_{i j}=0$. If $r_{i j} \leq R_{\min }, \tilde{a}_{i j}=1$. In both cases, $\tilde{a}_{i j}$ is independent of $R_{i}$ and $R_{j}$. Assume that $\left.\left.r_{i j} \in\right] R_{\min }, R_{\max }\right]$. Also assume that $R_{i} \leq R_{j}$ so that

$$
\tilde{a}_{i j}=\chi\left(r_{i j} \leq R_{i}\right) .
$$

If $R_{i}>R_{j}$, we apply a similar reasoning. Denote $k \in\left\{1, \ldots, d_{i}\right\}$ such that $r_{i j}=r_{i}^{k}$. If $k \leq k_{i}$, then, since $R_{i} \geq r_{i}^{k_{i}}, \tilde{a}_{i j}=1$ (by the 
assumption of the lemma). If $k \geq k_{i}+1$, then, since $R_{i}<r_{i}^{k_{i}+1}$, $\tilde{a}_{i j}=0$. In each case, $\tilde{a}_{i j}$ is independent of the value of $R_{l}$ for $l \in \mathcal{N}$. Weight $\tilde{a}_{i j}$ is independent of $R_{l}$ for $R_{l} \in\left[r_{l}^{k_{l}}, r_{l}^{k_{l}+1}\right.$ [, $l \in \mathcal{N}$. Thus, we may assume that $R_{i}=r_{i}^{k_{i}}$ and $R_{j}=r_{j}^{k_{j}}$ to compute $\tilde{a}_{i j}$. So, for $R_{i} \in\left[r_{i}^{k_{i}}, r_{i}^{k_{i}+1}\left[\right.\right.$ and $R_{j} \in\left[r_{j}^{k_{j}}, r_{j}^{k_{j}+1}\right.$,

$$
\tilde{a}_{i j}=\chi\left(r_{i j} \leq \min \left(R_{i}, R_{j}\right)\right)=\chi\left(r_{i j} \leq \min \left(r_{i}^{k_{i}}, r_{j}^{k_{j}}\right)\right) \text {. }
$$

We now introduce the main result of the section.

Proposition 22. The quantity $\mathbb{E}(\phi(\tilde{L}))$ has exact formulation :

$$
\mathbb{E}(\phi(\tilde{L}))=\frac{1}{\hat{R}^{n}} \sum_{k_{1}=0}^{d_{1}} \ldots \sum_{k_{n}=0}^{d_{n}} \prod_{i=1}^{n}\left(r_{i}^{k_{i}+1}-r_{i}^{k_{i}}\right) \phi\left(\tilde{L}\left(k_{1}, k_{2}, \ldots, k_{n}\right)\right) .
$$

To obtain the exact expression of $\mathbb{E}\left(\lambda_{2}(\tilde{L}(t))\right)$ or $\mathbb{E}\left(\left(\lambda_{2}(\tilde{L}(t))\right)^{2}\right)$, one needs to replace $\phi(\tilde{L})$ by these quantities in proposition 22 .

Remark 23. Proposition 22 gives an exact expression to the product $\mathbb{E}\left(\lambda_{2}(\tilde{L}(t))\right) \rho$. As a consequence, obtaining the maximum probability of flocking according to Theorem 12 comes down to maximizing this expression in function of $\rho$, which can be done using standard numerical methods. Once the optimal value of $\rho$ is obtained, proposition 22 also gives a value for $v_{\varepsilon}$.

Remark 24. If we assume that degrees $d_{i}$ are homogenous, the number of constant $\tilde{L}\left(k_{1}, k_{2}, \ldots, k_{n}\right)$ to be computed increases as $O\left(d^{n}\right)$ where $d$ is the average node degree. The computation can thus be done when $n$ is small. When $n$ is too large, one rather choose to compute an approximate value for $\mathbb{E}(\phi(\tilde{L}))$ using a Monte-Carlo method [MU49].

Proof of proposition 22. To stress that the matrix $\tilde{L}$ is a function of radiuses $R_{i}$, we use notation $\tilde{L}\left(R_{1}, R_{2}, \ldots, R_{n}\right)$. The radiuses $R_{i}$ for $i \in \mathcal{N}$ are independent random processes, therefore the probability density function of vector $\left(R_{1}, R_{2}, \ldots, R_{n}\right)$ is the product of the probability density functions of radiuses $R_{i}$ for $i \in \mathcal{N}$. Thus, we have

$$
\begin{aligned}
& \mathbb{E}(\phi(\tilde{L}))=\frac{1}{\hat{R}^{n}} \int_{R_{1}=R_{\min }}^{R_{\max }} \cdots \int_{R_{n}=R_{\min }}^{R_{\max }} \phi\left(\tilde{L}\left(R_{1}, \ldots, R_{n}\right)\right) d R_{1} \cdots d R_{n} \\
& =\frac{1}{\hat{R}^{n}} \sum_{k_{1}=0}^{d_{1}+1} \int_{R_{1}=r_{1}^{k_{1}}}^{r_{n}} \cdots \sum_{k_{n}=0}^{r_{n}} \int_{R_{n}=r_{n}^{k_{n}}}^{r_{n}} \phi\left(\tilde{L}\left(R_{1}, \ldots, R_{n}\right)\right) d R_{1} \cdots d R_{n} .
\end{aligned}
$$

We can then use lemma 21 so that

$$
\mathbb{E}(\phi(\tilde{L}))=\frac{1}{\hat{R}^{n}} \sum_{k_{1}=0}^{d_{1}}\left(r_{1}^{k_{1}+1}-r_{1}^{k_{1}}\right) \cdots \sum_{k_{n}=0}^{d_{n}}\left(r_{n}^{k_{n}+1}-r_{n}^{k_{n}}\right) \phi\left(\tilde{L}\left(k_{1}, \ldots, k_{n}\right)\right) .
$$

\section{B. Simulations}

In this section we use a simple 6-agent system to illustrate our theoretical results. The agents evolve in $\mathbb{R}^{2}$ and initially divide in two groups with velocities bearing opposite $x$ coordinates. The initial positions induce a connected communication network with positive probability. We wonder with which probability the group will overcome the initial conflict and eventually stay together achieving a flocking behavior. According to Theorem 12, our bound on the probability of flocking depends on the ratio $\frac{\tilde{2}_{2} \rho}{\|\delta(0)\|}$. The numerator is completely determined by the agents' positions (vector $x^{0}$ ) while the denominator is a function of the initial velocities (vector $v^{0}$ ). In order to explore the different outcomes of the system, we fix the initial positions and vary the initial velocities. Precisely, we choose $x_{x}^{0}=(-3.5,-2.3,-1.5,1.5,2.3,3.5)$ and $x_{y}^{0}=(1.5,0.9,0,0,0.9,1.5), \bar{R}=3.1, \hat{R}=2$ (which makes the initial interaction graph $G(0)$ connected when all communication radiuses $\left.R_{i}(0)=\bar{R}\right)$. We set a reference vector of initial velocity disagreement $d=\frac{1}{\sqrt{6}}(-1,-1,-1,1,1,1)^{T}$ so that $\|d\|=1$. We choose the $x$-coordinate of the initial velocity vector $v_{x}^{0}=\gamma d$ where $\gamma$ is the parameter we use to tune the system. We take the $y$-coordinate $v_{y}^{0}=c \mathbf{1}$ where $c>0$ is an arbitrary constant which does not influence the flocking outcome of the dynamics and serves only for visualization purposes. We have $\|\delta(0)\|=\left\|(I-J) v^{0}\right\|=\|\gamma d\|=\gamma$ so $\gamma$ represents the amplitude of the initial velocity disagreement.

Since $x^{0}$ is fixed, we can determine the optimal product $\mathbb{E}\left(\lambda_{2}(\tilde{L})\right) \rho$ for $\rho \in\left[R_{\text {min }}, R_{\text {max }}\right]$. The optimization gives $\rho_{\text {opt }}=$ 0.34 and $\mathbb{E}\left(\lambda_{2}\left(\rho_{\text {opt }}\right)\right) \rho_{\text {opt }}=0.018$. We choose the time step $\varepsilon=$ 0.2 as suggested in Remark 3 which gives a value $v_{\varepsilon}=0.0026$.

To illustrate the results, we ran some simulations. First, we chose $\|\delta(0)\|=0.9 \frac{\rho \tilde{\lambda}_{2}}{\sqrt{2}}$ which leads to a lower bound on the probability of flocking of 0.82 . Then, we chose $\|\delta(0)\|=10 \frac{\rho \tilde{\lambda}_{2}}{\sqrt{2}}$ for which Theorem 12 does not provide any information regarding the asymptotic behavior. Figure 1 presents snapshots of the evolution of the system for a realization of the random process for the two different choices of $\|\delta(0)\|$ exhibiting different outcomes. In the first case, the group asymptotically converges to flocking despite that the graph does not remain connected at all times. The second part of the figure presents a case where the initial velocity disagreement is too high and the two subgroups split apart, never reaching the flocking behavior.

\section{Parameter exploration}

We consider the system 1 with random communication radiuses as described in section V-B. Our goal is to explore the influence of the width of the communication range on the bound given by Theorem 12. To make the discussion more concrete, we assume that agents in system (1) represent robots using wifi communication. The initial positions are the ones described in section VI-B. The agents have the possibility to increase their maximal communication radius, but this comes at the cost of decreasing the minimal radius so that the average radius remains a constant. Using the notation defined in section $\mathrm{V}-\mathrm{B}$, this means that the radius diameter $\hat{R}$ is allowed to vary whereas the average radius $\bar{R}$ is fixed. We wonder whether this possibility may increase the critical threshold $\frac{\rho \tilde{\lambda}_{2}}{\sqrt{2}}$. This threshold is the limit on the initial velocity disagreement vector norm $\|\delta(0)\|$, beyond which no probability of flocking is guaranteed by Theorem 12 .

The answer depends on the choice of the initial mean communication radius $\bar{R}$. We illustrate this fact using two 

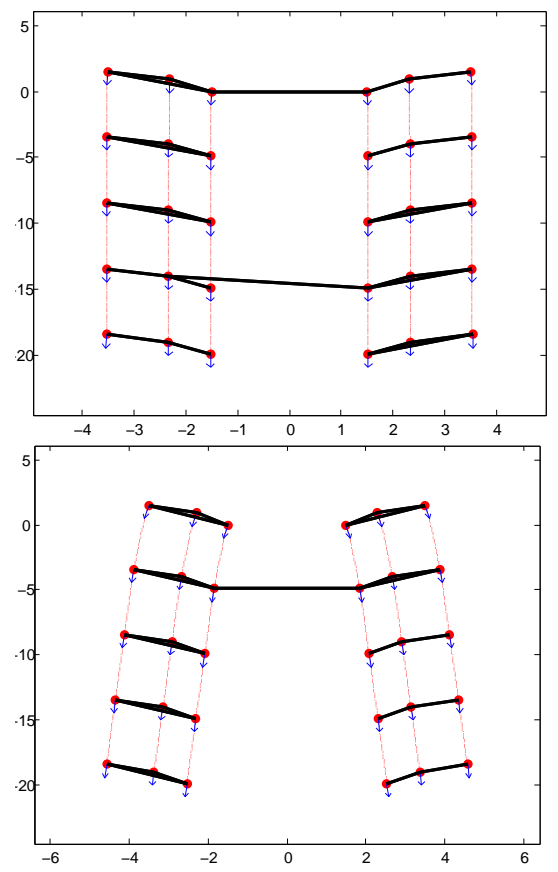

Fig. 1. The upper part of the figure presents an instantiation of the dynamics for $\|\delta(0)\|=0.9 \frac{\rho \tilde{\mu}_{2}}{\sqrt{2}}$. The lower part is an instantiation when $\|\delta(0)\|=10 \frac{\rho \tilde{\lambda}_{2}}{\sqrt{2}}$. The figure shows the trajectories of the 6 agents and their positions, velocities and connections for 5 specific times. The six extreme top red dots are the initial agents' positions. The blue arrows represent the velocities (their amplitude has been increased 2 times for visualization purposes). The black lines between dots represent communication links.

distinct values for $\bar{R}: 2.7$ and 3.1. If we assume that all radiuses $R_{i}(0)=\bar{R}$, in the first case, the graph $G(0)$ with $R_{i}(0)=\bar{R}$ is unconnected, whereas in the second case, $G(0)$ starts connected. Using these two mean radiuses, we then vary $\hat{R}$. For each couple $(\bar{R}, \hat{R})$, we computed the optimal authorized perturbation $\rho$ maximizing the product $\rho \tilde{\lambda}_{2}$. Figure 2 presents the results for the two different $\bar{R}$.

In the upper part of figure $2, \bar{R}=2.7$. We see that the probability of flocking is null for small $\hat{R}$ values, which comes from the fact that $\rho \tilde{\lambda}_{2}$ is null, this is consistent with the fact that the graph is disconnected when communication radiuses $R_{i}$ are close to 2.7 and thus $\tilde{\lambda}_{2}=0$. The increase in diameter $\bar{R}$ allows for larger $R_{i}$ values, which in turns gives a more connected graph on average (i.e. $\tilde{\lambda}_{2}$ is higher). The initial communication graph is also more robust, so that $\tilde{G}$ remain connected even for higher $\rho$ values, giving a higher $\rho_{\text {opt }}$ value. This example illustrates that when $\bar{R}$ is such that $G(0)$ is disconnected for small value of $\hat{R}$, the increase of $\hat{R}$ can only increase the bound for flocking, given in Theorem 12.

The lower part of figure 2 demonstrates that the previous comment does not hold for all $\bar{R}$ values. Here, $\bar{R}=3.1$ so that $G(0)$ is connected for small value of $\hat{R}$. As mentioned, the increase of the diameter $\hat{R}$ allows for higher $R_{i}$ values but also smaller ones. So that in the instance where a small decrease on $R_{i}$ is sufficient to disconnect $G(0)$, increasing $\hat{R}$ may lead to decreasing the average connectivity $\tilde{\lambda}_{2}$. In turn, this may decrease the lower bound on the probability of flocking. This is what is seen in the lower part of figure 2 , for small $\hat{R}$ values.

\section{Lower bound on the probability of flocking}
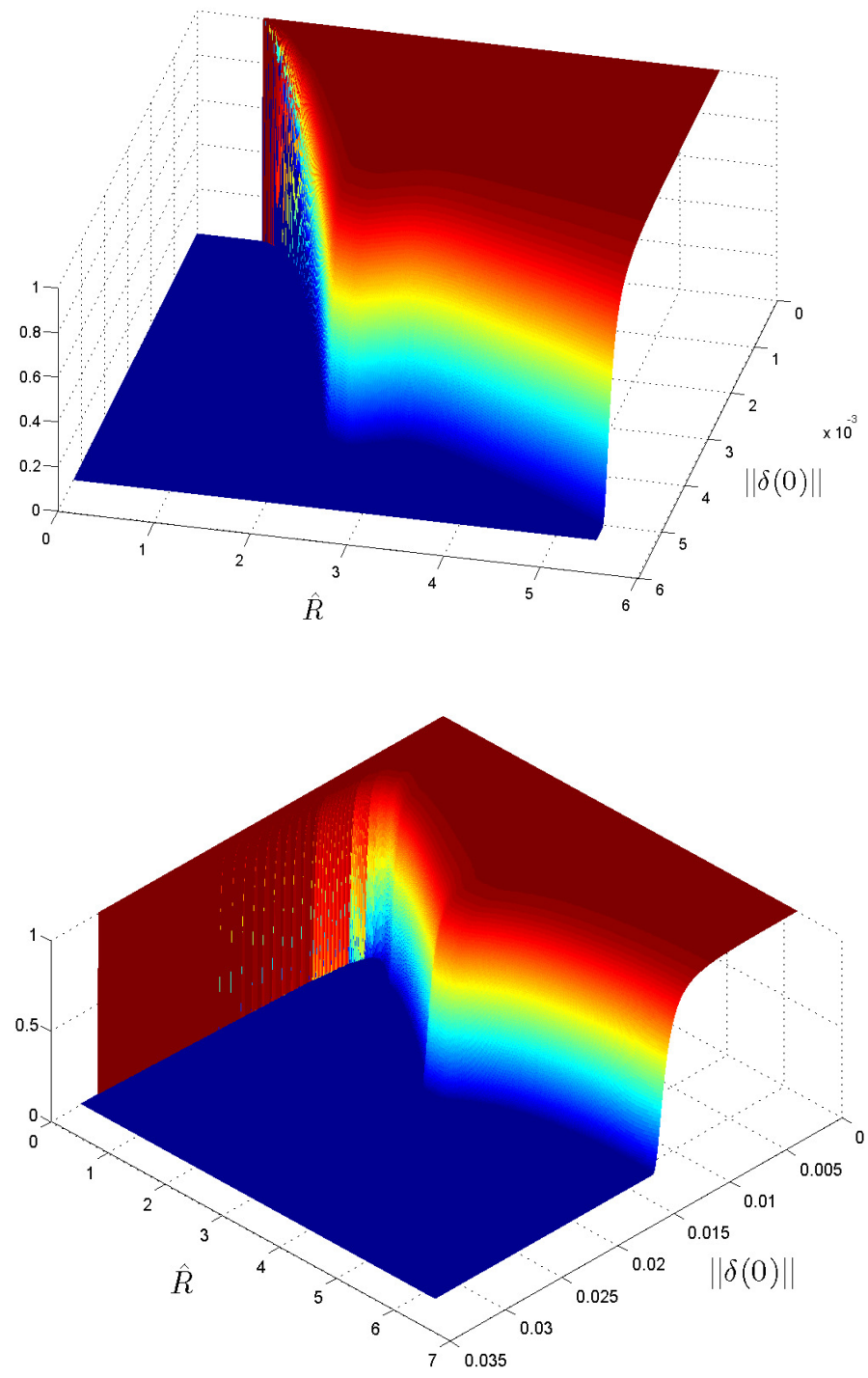

Fig. 2. The figure shows the lower bound on the probability of flocking (given by Theorem 12) in function of the communication diameter $\hat{R}$ and the initial velocity disagreement norm $\|\delta(0)\|$. The value are given after optimization of product $\rho \tilde{\lambda}_{2}$ in function of $\rho$. The upper part of the figure presents the case where $\bar{R}=2.7$ (see Section VI-A). The lower part is when $\bar{R}=3.1$.

In this instance, reducing the communication radius variance gives the highest bound on the probability of flocking.

Remark 25. Before closing the section on numerical validation, we comment the conservativeness of our results. Regarding the result we have obtained in the deterministic setting (Theorem 11), it can be shown (see [Mar12]) that the bound on the velocity disagreement vector is tight. This $i$ s, there exists a sequence of systems $\left(S_{n}\right)$ of $n$ agents whose largest $\|\delta(0)\|$ leading to flocking converges to the bound given in Theorem 11 when $n$ goes to $+\infty$. Regarding the stochastic case, the bound in Theorem 12 is not as tight as the one in Theorem 11. The reason why this is the case comes from the 
following intuition. We use $1-\varepsilon \mathbb{E}\left(\lambda_{2}(\tilde{L})\right)$ in order upper bound the convergence speed of $\|\delta(t)\|$. However, if $\varepsilon$ is small enough, the effect of communcations are averaged over time and $\|\delta(t)\|$ converges according to $1-\varepsilon \lambda_{2}(\mathbb{E}(\tilde{L}))$ instead. For instance, $\tilde{L}$ may correspond to a graph never connected but still connected on average; this gives $\mathbb{E}\left(\lambda_{2}(\tilde{L})\right)=0$ but $\lambda_{2}(\mathbb{E}(\tilde{L}))>0$. In this case, we expect $\|\delta(t)\|$ to converge to 0 but Theorem 12 would fail to predict it.

\section{Conclusion}

In this paper, we have considered a multi-agent system consisting of mobile agents with second-order dynamics, where the communication network is determined by a general metric rule. Our approach extends earlier work from Martin and Girard [MG10] and Martin et al. [MFJG12]. It links algebraic connectivity of the communication network to the speed of convergence towards consensus.

We have established a lower bound on the probability of velocity alignment depending on the initial positions and velocities of the agents. Our main contribution has been to propose a suitable bounding process to the original system. Our main theoretical result states that flocking occurs whenever the initial velocity disagreement is smaller than a threshold (formed with the robustness and the algebraic connectivity of the graph of the bounding process). This result allowed us to derived practical bounds for flocking for two distinct communication rules. In the first case, agents communicate when their distance is smaller than a certain fixed communication radius. In a second application, we have allowed the communication radiuses to be different for each agent and to vary randomly over time. The deterministic system is thus a limit case of the stochastic one. For the stochastic system, we showed that the probability of flocking can be made higher than any constant arbitrarily close to 1 by choosing an appropriate initial velocity disagreement or by setting the system time step small enough. Finally, we have investigated the impact of the communication radius mean and variance on the bound for flocking. The main interest of our approach is the possibility of ensuring flocking a priori. The condition can be easily verified through numerical computation.

One limitation of our approach is to require symmetry in the interactions. In fact, it is possible to assume other types of interactions which are not-symmetric. For instance, one may assume that agents communicate with their $m$ closest neighbors, with $m$ a constant parameter ; this is the so-called topological interaction rule. See [Mar13] for an analysis of the flocking behavior under topological interactions. Another way to break the symmetry is the following. The value of $\epsilon$ in system (1) can be viewed as the discretization time step of the equivalent continuous-time flocking system. This is why it is a constant. However, we may also think of $\epsilon$ as the susceptibility of one agent to be influenced by the rest of the group. Taking a small $\epsilon$ for some agents and a big one for others would mean that some agents are more stubborn than others. This leads to non-symmetric interactions. The consequence of this are explored for the first-order case in $\left[\mathrm{YAO}^{+} 11\right]$.
Before switching to different class of systems, we plan to improve the tightness of the bound by taking into account two facts: we will relate velocities with positions because two agents with opposite velocities have more chance to agree on their velocities if they point toward each other, than if they point away from each other. Also, a subgroup of agents with high connectivity is intuitively more inclined to agree on their velocities than a subgroup of low connectivity. Thus, agents belonging to a highly connected local neighborhood should be allowed higher initial velocities (see for instance [FM12]). Finally, assuming connectivity of the union of graphs rather than connectivity at all time is sufficient for flocking [BHT05]. Taking into account this fact may lead to a less conservative bound. However, only having connectivity on the union of graphs over some time interval would lead to a slower convergence of the velocity disagreement. Thus, it is not clear whether this approach would lead to a better bound.

Also, our general theoretical result providing a bound for flocking may be applied to many different metric communication rules such as the Cucker-Smale type [CS07].

Another possible extension to the present work is to derive the probability that the initial configuration realize the bound given in Theorem 7. This may be done assuming the positions and velocities are uniformly distributed in a region of the space. To do so, we could for use ideas from the random goemetric graphs literature [Pen03].

\section{REFERENCES}

[AOP10] D. Acemoglu, A. Ozdaglar, and A. ParandehGheibi. Spread of (mis) information in social networks. Games and Economic Behavior, 2010.

[BHT05] V. Blondel, J. Hendrickx, and J. Tsitsiklis. Convergence in multiagent coordination, consensus, and flocking. $c d c, 2005$.

[BW09] S. Bermudez and S. B. Wicker. Connectivity of finite wireless networks with random communication range nodes. In IEEE International Conference on Communications, pages 1-5, 2009.

[CB09] T. B. Curtin and J. G. Bellingham. Progress toward autonomous ocean sampling networks. Deep Sea Research Part II: Topical Studies in Oceanography, 56(3-5):62 - 67, 2009. AOSN II: The Science and Technology of an Autonomous Ocean Sampling Network.

[Cha09] Bernard Chazelle. The convergence of bird flocking. CoRR, abs/0905.4241, 2009.

[CM08] F. Cucker and E. Mordecki. Flocking in noisy environments. Journal de Mathmatiques Pures et Appliques, 89(3):278 - 296, 2008.

[CMKB04] J. Cortes, S. Martinez, T. Karatas, and F. Bullo. Coverage control for mobile sensing networks. Robotics and Automation, IEEE Transactions on, 20(2):243-255, April 2004.

[CS07] F. Cucker and S. Smale. Emergent behavior in flocks. tac, 52(5):852 -862, may 2007.

[DM09] F. Dalmao and E. Mordecki. Cucker-Smale Flocking Under Hierarchical Leadership and Random Interactions. ArXiv eprints, December 2009.

[FM12] M. Fiacchini and I.-C. Morrescu. Set theory based condition in lmi form for network topology preservation for decentralized control. In $c d c$, dec. 2012.

[FZ07] F. Fagnani and S. Zampieri. Randomized consensus algorithms over large scale networks. In Information Theory and Applications Workshop, 2007, pages 150-159, 2007.

[GR01] C. Godsil and G. Royle. Algebraic Graph Theory. Springer, April 2001.

[HM05] Y. Hatano and M. Mesbahi. Agreement over random networks. IEEE Transactions on Automatic Control, 50(11):1867-1872, 2005.

[IJ09] M. Ion and B. John. Convergence results for the linear consensus problem under Markovian random graphs. 2009. 
[JLM03] A. Jadbabaie, J. Lin, and A.S. Morse. Coordination of groups of mobile autonomous agents using nearest neighbor rules. IEEE Transactions on Automatic Control, 48(6):988-1001, 2003.

[KM07] S. Kar and J. M. F. Moura. Sensor networks with random links: Topology design for distributed consensus. IEEE Transactions on Signal Processing, 56, 2007.

[Mar12] Samuel Martin. Coordination et robustesse des systèmes dynamiques multi-agents. $\mathrm{PhD}$ thesis, Université de Grenoble, 2012.

[Mar13] Samuel Martin. Multi-agent flocking under topological interactions. submitted for publication, available at http://arxiv.org/abs/1311.6046, 2013.

[Mer94] R. Merris. Laplacian matrices of graphs: a survey. Linear Algebra and its Applications, 197-198:143 - 176, 1994

[MFJG12] S. Martin, A. Fazeli, A. Jadbabaie, and A. Girard. Multi-agent flocking with random communication radius. In acc, jun. 2012.

[MG10] S. Martin and A. Girard. Sufficient conditions for flocking via graph robustness analysis. In $c d c$, pages $6293-6298$, dec. 2010.

[Mor05] L. Moreau. Stability of multiagent systems with timedependent communication links. IEEE Trans. Automatic Control, 50(2):169-182, Feb 2005

[MU49] N. Metropolis and S. Ulam. The monte carlo method. Journal of the American statistical Association, 44(247):335-341, 1949.

[OSFM07] R. Olfati-Saber, J. A. Fax, and R. M. Murray. Consensus and cooperation in networked multi-agent systems. Proceedings of the IEEE, 95:215-233, 2007.

[Pen03] Mathew Penrose. Random geometric graphs, volume 5. Oxford University Press Oxford, 2003.

[PS07] M. Porfiri and D.J. Stilwell. Consensus seeking over random weighted directed graphs. IEEE Transactions on Automatic Control, 52(9):1767-1773, 2007.

[PT07] G. Picci and T. Taylor. Almost sure convergence of random gossip algorithms. In $c d c$, pages 282-287, 2007.

[Rey87] C. W. Reynolds. Flocks, herds, and schools: A distributed behavioral model. Computer Graphics, 21(4):25-34, 1987.

[TBA86] J. N. Tsitsiklis, D. Bertsekas, and M. Athans. Distributed asynchronous deterministic and stochastic gradient optimization algorithms. IEEE Trans. Automatic Control, 31(9):803-812, 1986.

[TJP07] Herbert G Tanner, Ali Jadbabaie, and George J Pappas. Flocking in fixed and switching networks. Automatic Control, IEEE Transactions on, 52(5):863-868, 2007.

[TN10] B. Touri and A. Nedich. On ergodicity, infinite flow and consensus in random models. Automatic Control, IEEE Transactions on, (99):1-1, 2010.

[TSJ06] A. Tahbaz-Salehi and A. Jadbabaie. On consensus over random networks. In $c d c$, September 2006.

[TSJ08] A. Tahbaz-Salehi and A. Jadbabaie. On consensus over random networks. IEEE Transactions on Automatic Control, 53(3):791795, 2008.

[TSJ10] A. Tahbaz-Salehi and A. Jadbabaie. Consensus over ergodic stationary graph processes. IEEE Transactions on automatic Control, 55(1):225-230, 2010.

[VCBJ $\left.{ }^{+} 95\right]$ T. Vicsek, A. Czirók, E. Ben-Jacob, I. Cohen, and O. Shochet. Novel type of phase transition in a system of self-driven particles. Phys. Rev. Lett., 75(6):1226-1229, Aug 1995.

[Wu06] C.W. Wu. Synchronization and convergence of linear dynamics in random directed networks. IEEE Transactions on Automatic Control, 51(7):1207-1210, 2006.

[YAO $\left.{ }^{+} 11\right]$ Ercan Yildiz, Daron Acemoglu, Asuman Ozdaglar, Amin Saberi, and Anna Scaglione. Discrete opinion dynamics with stubborn agents. Available at SSRN 1744113, 2011.

[ZTJP09] M.M. Zavlanos, H. G. Tanner, A. Jadbabaie, and G. J. Pappas. Hybrid control for connectivity preserving flocking. Automatic Control, IEEE Transactions on, 54(12):2869-2875, 2009.

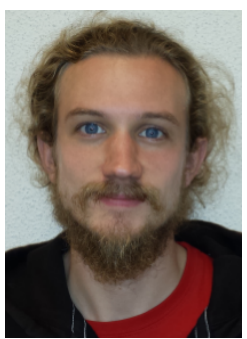

Samuel Martin received the Dip. Ing. from the Ecole Nationale Suprieure d'Informatique et de Mathématiques Appliquées de Grenoble, France, in 2009 and the Ph.D. degree in Applied Mathematics from the Universite de Grenoble, France, in September 2012. From January 2013 to August 2013, he was a postdoctoral researcher at the Large Graph and Networks group, Université Catholique de Louvain, Belgium. Since September 2013, he has been an Assistant Professor at the CRAN, Universit de Lorraine, France. His research interests include mutli-agent systems, consensus theory and applications to social networks.

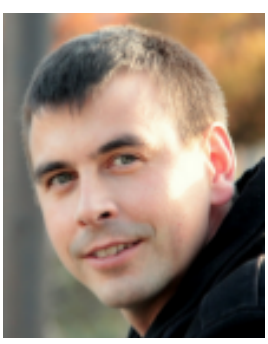

Antoine Girard received the Dip. Ing. from the Ecole Nationale Supérieure d'Informatique et de Mathématiques Appliquées de Grenoble and the Ph.D. degree in Applied Mathematics from the Institut National Polytechnique de Grenoble, France, in September 2004. From October 2004 to December 2005, he was a postdoctoral researcher at the Department of Electrical and Systems Engineering of the University of Pennsylvania and from January to August 2006, he was a postdoctoral researcher at the Verimag laboratory, Grenoble, France. In September 2006, he joined the faculty of Universit Joseph Fourier, Grenoble, France where he is an associate professor. His research interests deal with hybrid systems, distributed systems, hierarchical and symbolic control. He is a recipient of the George S. Axelby Outstanding Paper Award in 2009 and of the CNRS Bronze Medal in 2014.

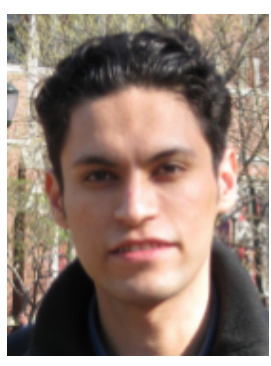

Arastoo Fazeli is a $\mathrm{PhD}$ candidate at department of Electrical and Systems Engineering, University of Pennsylvania. His research interests include stochastic processes, game theory and optimization. He his interested in applications to social and economic networks and finance. He has done research on option trading, viral marketing and pricing games in social networks and consensus

in random networks.

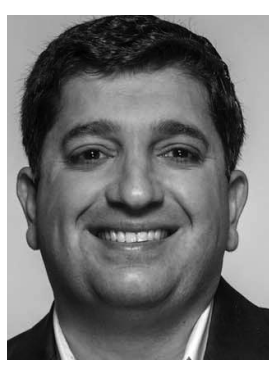

Ali Jadbabaie received the M.S. degree in electrical engineering from the University of New Mexico, Albuquerque, and the Ph.D. degree in control and dynamical systems (CDS) from the California Institute of Technology (Caltech), Pasadena, in 1997, and 2000, respectively. From October 2000 to July 2001, he was a Postdoctoral Scholar at the CDS Department at Caltech. From July 2001 to August 2002, he was a Postdoctoral Scholar at the Electrical Engineering Department, Yale University, New haven, CT. Since July 2013, he has been the Alfred Fitler Moore Professor of Network Science Department of Electrical and Systems Engineering, University of Pennsylvania His research interests are in real-time optimizationbased control with applications to Unmanned Aerial Vehicles, distributed coordination of mobile autonomous vehicles, and robust and optimal control. 